\title{
Memorable navigation: Assessing the influence of way-finding in spatial clustering
}

\author{
A. Pastor \\ 4 \\ Information Technologies, Multimedia and Telecommunications Department, \\ Universitat Oberta de Catalunya, Barcelona, Spain \\ alvaropastor@uoc.edu
}

\begin{abstract}
Navigating around an environment and remembering the events that took place within it are crucial cognitive abilities that have been linked to the Hippocampus and medial temporal lobes (MTL). Scene Construction Theory (SCT) has proposed that a function of the Hippocampus is the implicit and continuous construction of scenes to help prediction of upcoming environment. Scenes, as highly efficient means of packaging information, underpin in coordination with other brain regions, episodic memory (EM), spatial navigation, future thinking and perhaps even dreaming and mind-wandering. We inquired the conditions in which spatial contiguity of stimuli influences the organization of memory by examining spatial clustering (SC) phenomenon. In this research, an augmented reality (AR) system was used to test 14 participants in a spatially dependent memory task which assessed the SC differences between active navigators and passive spectators. We confirmed our hypothesis that navigators use spatial information as part of the retrieval process in free recall, as they tended to sequentially recall any two neighboring otherwise unrelated items. We also found a significant correlation between SC and correct recall performance supporting our second hypothesis. These results may be valuable for design of learning applications, especially dealing with large amounts of data. Research on Alzheimer's and other neurodegenerative diseases may also benefit from our approach. Future studies may assess the role of encoding and retrieval modality and participant's use of mnemonic strategies.
\end{abstract}

Keywords: Episodic memory Spatial clustering - Scene Construction Theory · Free Recall · Augmented Reality

\section{Introduction}

As described in Hume's Principles of Association [1], ideas enter into association with one another when they are contiguous in space and time. In the study of human memory, this contiguity effect has been found in studies of free recall, suggesting that a person is more likely to recall words together that are closer together in a list [2]. The contiguity effect appears relatively constant, and has been predicted to have long-term effects on memory according to researchers[3]. Whereas a massive body of experimental work has demonstrated the potent 
40 influence of temporal contiguity on the association of ideas, we know little about the conditions in which spatial contiguity of stimuli influences memory.

This study examined whether and how spatial navigation influenced the organization of memory by examining spatial clustering (SC) phenomenon and overall recall performance (ORF), using an ecologically valid experimental environment. Previous research has shown that the neural mechanisms of EM and navigation in real and mental space are fundamentally the same. It has been proposed that mechanisms of EM have evolved from mechanisms of navigation in the physical world. A clear parallel between allocentric navigation strategies and semantic memory, on one hand, and egocentric strategies and EM on the other 50 has been suggested. Importantly, studies have confirmed that the brain systems involved in guiding navigation, the Hippocampus and entorhinal cortex, are the 52 same that are activated in declarative memory tasks [4], and that the same mechanisms that define unique positions and their relationships in a map can be used to mediate memory for events, objects and living things [5]. Also, the Hippocampus - entorhinal cortex system has the anatomical and physiological properties Construction Theory (SCT) has pointed that a function of the Hippocampus is packaging information, to help prediction of upcoming environment [6-9]. Therefore, we found reasonable to expect that, when spatial information is available at encoding, Hippocampus would form a scene associating the encountered item 62 and spatial context, and that this composite scene would tend to be reinstated at recall. This reinstatement would not only provide the phenomenological expe64 rience of remembering, but also help to cue other memories experienced within the same or related spatial contexts.

From this literature overarching questions emerged: Can we derive learning strategies that profit from the mechanisms of scene dependent memory? Can design criteria for learning applications? We based our approach on prior studies a spatiotemporal context and that this context becomes reactivated when the 72 experience is recalled $[2,10-12]$. In this sense, this study aimed to advance our understanding of the conditions in which spatial contiguity of stimuli influences 74 organization of EM, and if spatial organization of memory correlates to increased performance.

76

If spatial navigation was essential for the formation of the spatiotemporal context of memory for a given stimulus, we hypothesized that, subjects who navigate an environment would exhibit a significant tendency to consecutively recall neighboring items presented therein. We compared these active performances to that of passive spectators of the same journey of presentation of target items. We also hypothesized that participants showing higher rates of spatial clustering 82 (SC) for a navigation task would present enhanced memory performance. 
This article summarizes the findings and updates the interpretation of the second pilot experiment of the Master Thesis Assessing the influence of spatial clustering in free recall [13].

Following a between subjects design we conducted a pilot experiment in which participants were presented with items in two spatially dependent conditions: Way finding group in which participants were presented via Augmented Reality (AR) with visual items upon arrival at various real-world locations, compared to a guided navigation group in which participants passively spectated the navigation recording of a corresponding matched-pair, featuring a 3D representation of the traversed environment.

To explore the conditions in which spatial contiguity of stimuli influences the organization of memory, we assessed the performance differences between active navigators and passive spectators in a spatially dependent memory task, using behavioral data, spatial clustering factor (SCF), and overall recall factor (ORF) as scores in Immediate (IFR) and delayed free recall (DFR) 24 hours after trial. Regarding the first hypothesis, this study found significant differences in SC between navigators and guided participants. We also found a significant correlation between $\mathrm{SC}$ and correct recall performance supporting our second hypothesis.

Our AR framework helped to evidence functioning of recall dynamics in both the guided and way-finding conditionsin a ecologically valid and systematic way. We suggested further studies to clarify participants' use of mnemonic techniques and the role of stimuli modality at encoding and retrieval. Advances in this field may benefit the design of AR learning applications and spatial data interaction paradigms, as well as research on cerebral aging and neurodegenerative conditions such as Alzheimer's disease.

\section{Background}

\subsection{Episodic memory}

Episodic memory [14] refers to autobiographical memory for events constructed from its what, when, where components, linked together in a coherent spatiotemporal experience. A key feature of episodic memory is its unique role to allow the individual to mentally travel back into her personal past $[15,16]$. The precise details that characterize episodic memory have been debated, but there is consensus that time, space and sense of self are key elements [17-21]. From research carried out using functional magnetic resonance imaging (fMRI) we know that a distributed and highly consistent network of brain regions supports memory for past experiences $[21,22]$. It has been suggested that episodic memory in the Hippocampus is formed by combining spatial information from the medial entorhinal cortex $[23,24]$ with non spatial information from the lateral entorhinal cortex [25,26]. Imaging studies have also illuminated the contributions of distinct prefrontal regions to encoding and retrieval [27-29]. Episodic memory is affected by aging processes [30], and there have been findings of sex differences 
in its performance [31,32], but results are still inconclusive [33]. Recent studies have helped to establish that these neural systems also support the capacity to imagine and to simulate episodes expected to occur in the future $[15,34-36]$. as prospection [37] and by others as episodic future thinking [38-40]. Consistent with this approach, neuroimaging studies focused on the brain activity during the construction of past and future events, have revealed regions exhibiting common activity, which included the left portion of the Hippocampus and the right occipital gyrus, especially during the elaboration phase, when participants are focused on generating details about the remembered or imagined event $[41,42]$.

\subsection{Theories of hippocampal function}

For decades, the Hippocampus and MTL of the mammalian central nervous system have been structures closely associated with memory [43-46]. Indeed, studies on Amnesiac and hippocampal excised patients has shown that the Hippocampus is essential for acquiring and consolidating new knowledge about events, people, and places. Several theories regarding the role of the Hippocampus in memory have been proposed over the years. All regard the Hippocampus as being critical for EM, but there are key differences between its mediator or storage roles.

Declarative Theory $[\mathbf{4 7}, \mathbf{4 8}]$ The Hippocampus, acting together with other medial temporal lobe regions, is crucial for all forms of consciously accessible memory processes (episodic and semantic, recollection and familiarity) for a timelimited period. Ultimately all memories are consolidated to neocortical sites.

Multiple-Trace Theory [49] The Hippocampus, together with other medial temporal lobe regions, is crucial for the acquisition of episodic and semantic memories. The recollection of episodic memories remains dependent on the Hippocampus for the duration of one's life and becomes more resistant to partial damage with repetition and/or rehearsal, whereas semantic memories become independent of the Hippocampus and are stored in other brain regions over time.

Dual-Process Theory $[\mathbf{5 0}, \mathbf{5 1}]$ The Hippocampus is crucial for episodic recollection of the contextual details of an event. Familiarity-based recognition processes are subserved by other medial temporal lobe regions. Recollection is required for the associative recognition of non-unitized items.

Cognitive-Map Theory [52] A primary role of the mammalian Hippocampus is to construct and store allocentric representations of locations in the environment to aid flexible navigation, for example, from a new starting position. In humans these predominantly spatial processes have evolved to support the spatio-temporal context of episodic memories.

Relational Theory $[\mathbf{5 3}, \mathbf{5 4}]$ Attempts to unify the declarative and spatial functions of the Hippocampus, suggesting a broader characterization including both functions: that the Hippocampus encodes relational information that can be flexibly applied to new situations. Thus it claims that the Hippocampus allows 
the flexible association of information in neocortical modules that could not oth-

\section{Navigation}

erwise communicate. This enables the relations between elements of a scene or event to be retrieved or used for inference in novel situations, in addition to retrieval of the elements themselves. The Cognitive-Map Theory can be subsumed as a special case of spatial relational processing.

Scene Construction Theory (SCT) [6-8] SCT suggests that a function of the Hippocampus is the implicit and continuous contruction of scenes to help prediction of upcoming environment. It does not suggest that the Hippocampus is solely responsible for EM, future thinking, and spatial navigation, but rather that the Hippocampus supplies the crucial ingredient of scene construction. SCT was originally proposed following the observation that patients with hippocampal damage and amnesia are unable to imagine scenes. Scenes are a highly efficient means of packaging information, that underpin and support -in coordination with other brain regions- EM, spatial navigation, future thinking and perhaps even dreaming and mind-wandering. Evidences from this hippocampal scene construction activity come from Boundary extension (BE) studies. BE is a robust and consistent cognitive phenomenon where we erroneously remember seeing more of a scene than was present in sensory input [55]. Accumulating evidence obtained by a variety of paradigms $[56,57]$ has shown that BE depends on the hippocampal ability to construct scenes. Predictive scene extension processes have been shown attenuated in hippocampal damaged patients, as they significantly reduced BE relative to matched controls across a number of independent measures. These data in addition to the original scene construction findings $[6-$ 8], support the idea that the primary function of the Hippocampus may not be mnemonic [58] but may instead be to predict the nature of the world beyond the immediate sensorium [9].

Navigating is the cognitive process of planning and following routes to travel from the current location to a target location. It involves way-finding, a fundamental human cognitive function, and motoric functions. Way-finding in complex environments relies on complex multisensory perceptual processes, from idiothetic and allothetic sources, and on memory [59-61]. When humans navigate, learn about the environment, by receiving spatial information from multiple sensory cues which are to be stored and used to guide subsequent navigation behavior. Consistent with Tolman's description of a cognitive-like map of the environment, the conception common to O'Keefe [52] and most researchers on cognitive maps, is that the Hippocampus mediates a holistic representation of space [62]. It has been shown that the spatially selective firing properties of rat hippocampal place cells are controlled by the salient sensory cues in the environment [63]. Research by Siegel and White [64] has provided a description of the process of the construction of a cognitive map as a person becomes familiar with an environment. A cognitive map begins as a set of landmarks, then it is followed by a representation of the routes, meaning the sequence of actions needed to get from one 
location to another. Finally, these sequences of actions or routes are united into an internal representation of the environment that is similar to a map, called a survey representation, which is useful because it can be used to generate novel routes between two locations within the environment. The resulting map would be Cartesian in that it provides metrics for the representation of distances and angles between the relevant stimuli. At the physiological level, a place cell reflects as a pointer, the occurrence of the organism at a particular coordinate position within the map. The formation of numerous independent maps in the Hippocampus has obvious advantages for memory formation, as it allows the network to store new representations in a manner that minimizes interference with previously stored memories. The orthogonal nature of hippocampal representations is expressed in its ability to remap between experiences and environments. Even minor changes in the configuration of landmarks or the motivational context can completely alter the firing pattern of the hippocampal cell population. As a result, each environment can be represented by a unique combination of active cells.

\subsection{Navigation Strategies}

Human navigation is based on two interlinked strategies for encoding and representation of the spatial environment $[65,66]$.

Allocentric Allocentric navigation is a mechanism that uses the spatial relationships among landmarks in order to define actual location in the environment and to provide static position information in a reference frame. Space representations in this system, may be updated frequently by changes in the configuration of multi sensory inputs.

Egocentric navigation A system which requires active movement of the body, and computes the distances and coordinates based on integration of motion and knowledge of previous positions, thus producing the spatial metric needed for the estimation of distances between landmarks [67]. To that end, egocentric navigation system relies on locomotion speed, elapsed time, head direction and the initial reference position. Allocentric and egocentric navigation always work together, but the availability of external landmarks may determine whether allocentric or egocentric strategies dominate.

\section{$3.2 \quad$ Way-finding}

A goal-seeking behavior, using allocentric and egocentric mechanisms, where the navigator must explore and fix the coordinates of his actual location, and compute the difference between his present coordinates and the goal coordinates to obtain the difference vector specifying the direction and distance to move from its current location to its goal $[68,69]$. 


\subsection{Neural substrate supporting spatial navigation}

Navigating around an environment is a crucial cognitive ability that has been linked to the Hippocampus and medial temporal lobes. While processing of spatial scenes involves the paraHippocampus, the right Hippocampus appears particularly involved in memory for locations within an environment, with the left Hippocampus more involved in EM, context-dependent autobiographical memory $[70,71]$.

Place Cells [72]. Fire selectively when the animal is in one particular region of the environment. It doesn't depend on the direction the animal is facing [62]. Firing persists in the dark [73], ruling out an explanation based on simple visual stimuli correlated with place. Different place cells fire in different environmental locations. The place cells are driven by a combination of perceptual inputs and self-motion information, either of which can individually update the place-cell representation. Once established, place cells can have the same firing pattern for months [74]. The firing patterns can persist even when all of the spatial cues are removed. Besides responding to incoming perceptual information, place cells are also driven by self-motion signals (proprioceptive, vestibular and reafferent signals from intended movements), which indicate the location of the animal on the basis of its own movements. There is an extensive literature describing characteristics of place cells in dorsal hippocampal area CA1 $[5,75]$. Less is known about the place code in other medial temporal lobe structures. It is known that there are place cells in the entorhinal cortex [76,77], a region of cortex that provides input to the Hippocampus proper.

Grid cells $[\mathbf{2 3}, \mathbf{7 8}]$. Grid Cells do not represent particular locations in the environment. Instead, they tile the environment, and are re-used over and over, like the intersections on graph paper. Grid cells have different periodic spacings, and it is believed that collectively, they form a universal, flexible code for mapping otherwise undifferentiated navigable space. Grid cells have multiple firing fields that span the entire available space in a periodic hexagonal pattern, which provides a metric to the neural representation of space. This type of cells system is present throughout the medial entorhinal cortex and in the pre and parasubiculum [79]. The correlation structure of grid cells is preserved during sleep periods [80].

Head direction cells [81] Their firing represents the direction in which the animal is heading, independent of its current location and the firing direction is controlled by self-motion and perceptual information. Head-direction cells show pattern completion and accommodation for missing cues, and probably set the environmental orientation of the responses of place cells. When stimulated, these neurons fire at a steady rate not showing adaptation, but decrease back to their baseline rates as the animal's head turns away more than 45 degrees from an specific direction [82]. These cells are found in many brain areas, including the post-subiculum, retrosplenial cortex, the thalamus (the anterior and the lateral dorsal thalamic nuclei), lateral mammillary nucleus, dorsal tegmental nucleus, striatum and entorhinal cortex [83]. 
Border Cells. O'Keefe and Burgess [84] observed that the medial entorhinal cortex contains a smaller number of border cells, which line up in specific orientations along specific geometric boundaries of the environment. These cell types were all discovered in rats, but place cells, grid cells and head direction cells have since been found in other species. Border cells may assist in the assessment of the allocentric distances by triangulation and perhaps in scaling the grid size to accommodate to the size of the discoverable environment [85].

\section{Space and memory connection: Spatial Clustering}

Most theories of memory search assume that the just-recalled item, and its associated semantic and contextual information, forms part of the retrieval cue for the next response. For instance, temporal clustering effects have been be observed to the extent that recalling an item retrieves its associated temporal context, as this information will be similar to the contexts associated with neighboring list items [3,12]. Similarly, SC effects should be observed to the extent that recalling an item retrieves its associated spatial features, as this information would be similar to the spatial features associated with spatially proximate items. Assuming that spatial attributes form an integral part of the item representations encoded in memory, it is reasonable to expect to see SC even in tasks where participants are not instructed to encode the spatial context of the to-be-learned items and where retrieval makes no explicit reference to the spatial characteristics of the items.

The key properties of EM involve binding disparate and often arbitrary details together into a coherent event and the recollection of self-centered past experiences in the context of time and space in which the events occurred [8].

Theories of EM suggest that memory encoding and retrieval are facilitated by spatiotemporal context: a continually updated representation of location in space and time. The Hippocampus appears to have neurons that are specialized for encoding both spatial and temporal information. Hippocampal place cells fire at specific locations within an environment [79], and recently documented hippocampal time cells fire at specific temporal intervals between distinct events [86]. Recent studies seek to examine the role of spatial context in human EM retrieval through a hybrid spatial and EM task. Miller et al. [87] identified place cells as neurosurgical patients performed a delivery task which required them to deliver items to different stores in a virtual town. Following this navigation task, patients were asked to freely recall the items they had delivered. Place cell activation patterns during the navigational task were then compared to the subsequent activation patterns that occurred during the free recall task. Miller found that neural activity during the retrieval of each delivered item was similar to the neural activity associated with the location where that item was encoded. These findings demonstrate context-specific reinstatement of place-responsive cell activity at the time of recall, supporting theories that implicate contextual reinstatement as the basis for memory retrieval [87]. Kahana and others [88] have found neural evidence for contextual reinstatement in humans. After studying a 
list of 20 words and performing a brief distraction task, a participant recalls as

\subsection{Hypotheses}

366

\section{Method} time span. prone to higher ORFs.

many words as he can remember, in any order. ECG activity is recorded during each study and recall event. Participants exhibiting stronger neural signatures of context reinstatement also exhibited more pronounced temporal contiguity effects [89]. Another example is the [90] findings on the reactivation of encodingrelated brain activity during memory retrieval. Howard et al [91] analyzed the firing rates of ensembles of individual neurons as neurosurgical patients implanted with hippocampal depth electrodes performed a continuous recognition memory task. They found that the ensemble similarity of neural activity when a photograph was repeated was similar to the neural activity recorded during the encoding of neighboring photos.

Following a between subjects design we conducted a pilot experiment in which participants were presented with items in two spatially dependent conditions: Way finding group in which participants were presented via AR with visual items upon arrival at various real-world locations, compared to a guided navigation group in which participants passively spectated the navigation recording of a corresponding matched-pair, featuring a 3D representation of the traversed environment. We aimed to avoid semantic and temporal clustering strategies between subjects by presenting with identical target items in the same inter stimuli

We developed an AR system that enabled users in the way-finding group to freely explore Poble Nou Central Park where they were presented with virtual items upon arrival at various real-world locations. Our AR system navigation recording features, used to replay a $3 \mathrm{D}$ representation of the way-finding sessions to the matched-paired participants in the guided condition. We measured ORF scores in IFR and DFR 24 hours after trial. We calculated SCF from the logged navigational data. Comparisons were made regarding SCF in the two experimental conditions. We inquired whether subjects higher SCF scores were more

The study was based on prior studies that show that recall dynamics is guided by item and context similarity [92], that SC exists in a two-dimensional environment [93], and that the EM system associates each new experience within a spatiotemporal context and that this context becomes reactivated when the experience is recalled $[10,87,94]$.

We reasoned that the spatial conditions in which an item is experienced would form a composite scene for the item, even if the item itself had no spatial information associated with it prior to the experiment. In this sense, when a memory is retrieved, its spatiotemporal context is partially reinstated. This reinstatement would not only provide the phenomenological experience of remembering, 
but also help to cue other memories experienced within the same or related spatiotemporal contexts. Therefore, in a task where participants were instructed to navigate around an environment and encode each object in a specific real-world location, it was reasonable to expect that recalling an item would reinstate its spatiotemporal context including nearby items in SC. In the case of being guided as static spectators, there would be less available spatial information at encoding time of the EM scene, thus, other clustering strategies (temporal, categorical) would organize recall.

We hypothesized that participants in the way-finding group would exhibit a significant difference to consecutively recall items presented in spatially proximate locations when compared to passive spectators. We also hypothesized that participants showing higher rates of SC would also show better memory performance. Overall, when participants recalled an item, we expected that the item will evoke its spatial context and thus enhance retrieval of unrelated items experienced in nearby spatial locations.

\section{$5.2 \quad$ Subjects}

We interviewed 29 volunteers from Universitat Pompeu Fabra at Poble Nou Campus in Barcelona. We then selected 7 matched pairs in terms of sex and previous knowledge of the experimental location. 14 participants (2 female) aged from 19 to 33 agreed to take part in this study and were assigned each to one of two groups, way-finding condition and guided condition. All participants reported user level experience with AR systems. All were right handed and none wore corrective lenses during the study.

Way-finding Condition Participants were required to explore the physical environment, deciding by themselves the path to follow, using a handheld AR system, which presented 17 virtual landmarks available only for one time display. In our AR system, way-finding was supported by adding virtual landmarks at selected positions of the physical space and rendering target items upon subject arrival.

Guided Condition Participants are presented with a realistic 3D representation of the Poble Nou Central Park, that replayed a session of the way-finding Condition. Participants watched the entire navigation for the same amount of time, with equal inter stimuli time span, and have the target items presented in similar conditions. We take into consideration that previous studies [87] have achieved SC by playing a video game portraying a detailed 3D model.

\subsection{AR system}

In order to convey a realistic and ecologically valid spatial experience, we developed an AR system using Unity software which enabled to overlay virtual information in real world positions according to participants' spatial coordinates (GPS) and orientation. This system was installed on an Apple iPad model MD369TY/A with 2048 x 1536 pixels visual display and used the built-in GPS 

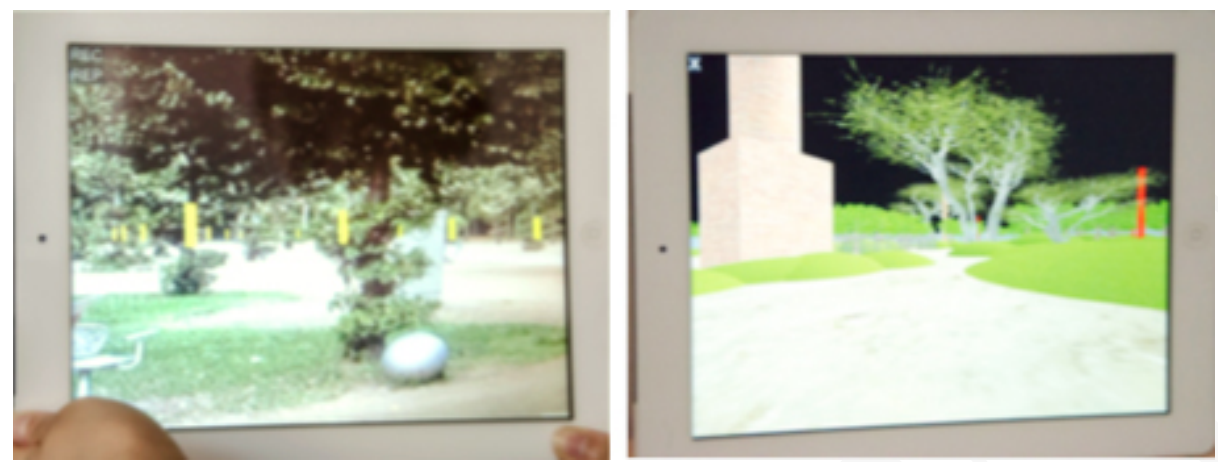

Fig. 1. Overview of two experimental conditions. The AR paradigm used for the WayFinding condition in the left image. A 3D virtual representation of the journey used for the guided Condition.

receiver. The AR system allowed us to present to participants, 17 virtual landmarks distributed over the physical space. This approach enabled participants to decide the path to follow, navigate and find their way through the space. Each time a participant arrived at the coordinate-defined physical location where the virtual landmark was placed the system presented a virtual target item on the handheld device display. Our system recorded all position and orientation transformations, and user interactions with target items during each experimental session, in order to be replayed for each of the guided condition participants with identical order, visual spatial information, and inter stimuli time span.

Location. Our study took place at Poble Nou Central Park, designed by architect Jean Nouvel and built in 2008. Our study was conducted in a 170 meter long by 60 meter wide area, comprising different landscape and architectural elements, such as gravel, ground, grass and cork flooring, an area with salient trees, two wooden huts, a brick chimney, and several children playground areas. Each of these features was accurately rendered in our 3D representation of the environment.

Virtual landmarks. 17 colorful virtual landmarks, equivalent size of 7 meter height and half a meter diameter cylinders, were placed in AR related to realworld locations. Each indicated a three meter equivalent radius interactive area where the user found a target item upon arrival. Virtual landmarks held no noticeable distinction from each other. The layout of the park and the location of the virtual landmarks was identical for every participant.

Target items. 17 items from the Moreno-Martinez and Montoro [95] alternative to Snodgrass \& Vanderwart [96] object image databank were selected to be presented to participants. These pre validated pictures were semantically significant everyday objects, rendered in a realistic appearance in $1024 \mathrm{px}$ wide by $768 \mathrm{px}$ height and $72 \mathrm{ppi}$ on the AR visual display, with item name captioned in $60 \mathrm{px}$ Sans Serif type. Any of the 17 target items only became visible for a 5 second 
${ }_{444}$ period when the participant physically walked into any of the three meter radius interactive areas indicated by the virtual landmarks.

446 Real - Virtual Landmark connections. We chose to locate each virtual landmark near existing architectural landmarks found at Poble Nou Central Park.

${ }_{448}$ Target items and physical landmarks held no semantical relation, as virtual target items were randomly allocated.
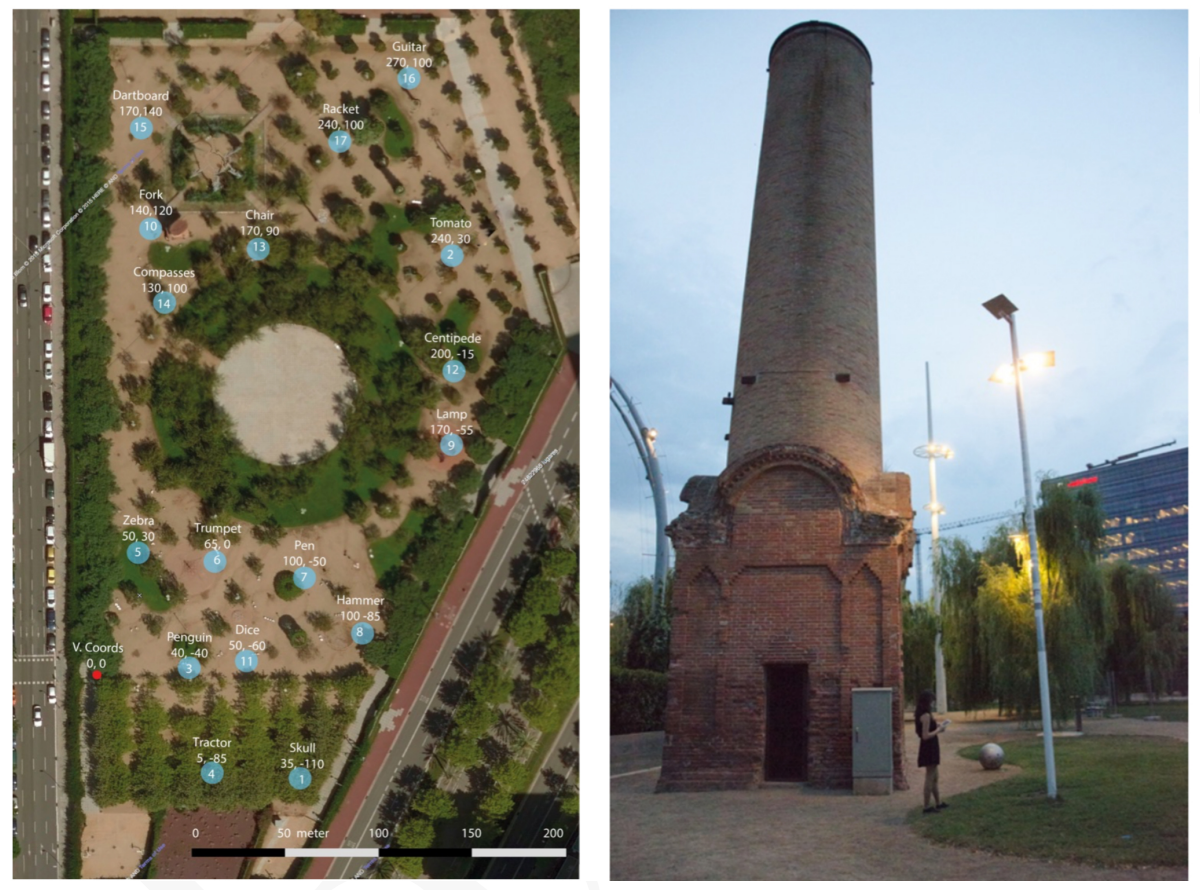

Fig. 2. Left, an orthographic image from the experimental location, and overlay of virtual landmarks and target items. Left, Virtual target items where distributed near existing architectural landmarks at Poble Nou Central Park.

\subsection{Procedure}

Before the start of each session, participants were given written instructions explaining the procedures to take place, and asked to give signed consent in order to begin the trial. Then, participants were guided through a 2 minute familiarization process with the AR system. Finally, participants were led to the trial starting position, defined at latitude 41.407221 and longitude 2.199322 which was set to be position $\mathrm{X}=0, \mathrm{Y}=0$ in our fixed coordinate system across trials. Maximum duration of the procedure was 35 minutes. 
Way-finding condition. The participants in the way-finding condition were asked to physically explore the real park environment during a maximum of 20 minutes using a hand-held device that allowed virtual landmarks and target items to be presented in AR. All 17 virtual landmarks are visible from the start, indicating real world locations where users were to find virtual target items.

Guided condition. In the Second Pilot Experiment, participants sat down at local coordinates $\mathrm{X}=0, \mathrm{Y}=0$, and were presented with a realistic 3D representation of the corresponding recorded session of a way-finding journey. Participants watched the entire navigation for the same amount of time and inter stimuli time span, and had the target items presented using the same display and in similar viewing conditions.

\subsection{Data Analysis}

Immediate and Delayed Free recall Free recall tasks allowed us to measure the way participants organize learned items [97-99]. EM was investigated by visually presenting items for study, and then asking participants to recall the items. Using a method for measuring SC modified from previous studies [87] we could quantify the way in which people transition from one recalled item to the next. For that reason, participants from both way-finding and guided conditions completed an IFR task, in which they were asked to verbally report as many recalled items as possible, in any order, for 90 seconds. 24 hours past from the experimental procedure, each participant was called by telephone to be questioned about the target items they were able to remember in free recall during 90 seconds in order to complete a DFR task. Recalled items were considered valid only if reported once and if they were actually presented to participant.

Spatial clustering factor. To determine whether the spatial proximity between items had an effect on the order in which items were recalled, we compare the SCF for each recalled item by each participant in the way-finding condition, to similar data from the guided condition. In order to establish a SCF for each of the reported recalled items, both in IFR as in DFR tasks for all participants, we compare the distances between a recalled item position and the position of the next recalled item. We used a custom version of the Haversine formula implemented in Python programming language. Then, we compared this measurement to the distance between a recalled item position and each of the available possibilities in the target item pool. A SFC of 1 was assigned to the most proximal locations between any given pair of items in the entire pool of items, whereas all other transitions between locations will receive proportional SFC values with 0 as a lower bound.

Spatial clustering and overall recall performance. To determine the impact of SC in overall recall performance, we first calculated the ORF of each session by defining the percentage of valid items recalled from the total of presented items. Then we normalized each percentage value to decimal range. To obtain an ORF of 1, all presented items during a session would have had to be recalled. 
At this point we conducted a Correlation Test between the ORF and the SCF average of each session to assess the influence of SC on recall performance.

\section{Results}

The sample for the Second Pilot Experiment consisted of 14 subjects ( 2 females) paired-matched and distributed in two groups, the way-finding and guided conditions. The mean age of participants equaled 25 years (average $\mathrm{SD}=1.1$ ). From the 17 target item pool that the procedure asked to be found, participants in the experimental condition scored a mean of 11,25 in target item findings. Results for the Kolmogorov-Smirnov test for normality indicated that the SCF distribution in way-finding deviated significantly from a normal distribution for IFR $(\mathrm{p}<.001)$ and DFR $(\mathrm{p}=.024)$. We then conducted Kolmogorov-Smirnov test for normality for ORF scores, which indicated that the ORF distribution did not deviate significantly from a normal distribution $(\mathrm{p}=.200)$.

Comparing SC in way-finding and guided conditions. A Mann-Whitney 2 tailed U test was conducted to compare the SCF for way-finding and guided conditions in both IFR and DFR tasks. We found statistically significant differences in both in IFR $\mathrm{U}=561, \mathrm{p}<.001$ and in DFR $\mathrm{U}=453.5, \mathrm{p}=.036$.

Correlations between SFC and Memory Performance A Pearson productmoment correlation coefficient was computed taking the complete set of free recall tasks to assess the relationship between the OFR scores obtained in each session and a SFC average of each session. We found a positive correlation between the two variables $\mathrm{r}=.499 ; \mathrm{p}=.007$. A Pearson product-moment correlation coefficient was computed considering only the DFR results to assess the relationship between the scores of recalled items obtained in each session's OFR and a SFC average score. There was a significant correlation between the two variables $\mathrm{r}=.607 ; \mathrm{p}=.021$. Computing a Pearson product-moment correlation coefficient only considering the results from IFR we found no statistically significant correlation between the two $\mathrm{r}=.108 ; \mathrm{p}=.497$.

\section{Discussion}

In recalling a list of previously experienced items, evidence has shown that humans organize their responses on the basis of the items' semantic and temporal similarities. This study aimed to shed light on the conditions in which spatial contiguity of stimuli influences the organization of memory.

If spatial navigation is essential for the formation of the spatiotemporal context of memory for a given stimulus, we hypothesized that, subjects who navigate an environment in the way-finding condition would exhibit a significant tendency to show SC in consecutively recalling neighboring items presented therein.

We also hypothesized that participants showing higher rates of SC during a navigation task would also show enhanced memory performance. 
Following a between subjects design we conducted a pilot experiment in which participants were presented with items in two spatially dependent conditions: Way finding group in which participants are presented via AR with target visual items upon arrival at various real-world locations, and a guided condition in which participants passively spectate the navigation recording of a corresponding matched-pair from $\mathrm{X}=0, \mathrm{Y}=0$ local coordinates of the same environment. We assessed the performance differences between active navigators and passive spectators measured in SFC and ORF using behavioral data and scores obtained via IFR and DFR.

Wayfinding significantly increased SC Regarding the first hypothesis, we evaluated whether a correlation between $\mathrm{SC}$ and navigation paradigm existed and found significant differences in SC favoring wayfinders. We found that navigation played a crucial role in the formation of context-based memories. As such, the target items encountered during navigation became associated with their spatiotemporal context at encoding. And when this memory was retrieved, the spatiotemporal context of the encoding episode was also reinstated. This reinstatement not only provided the phenomenological experience of remembering, but also helped to cue other memories experienced within related spatiotemporal contexts. We suggest the need for future research to address the roles of decision making and sensorimotor components of navigation and their contribution to SC.

SC significantly increased ORF For our hypothesis regarding enhanced memory performance, we found a significant positive correlation between SC and ORF $\mathrm{r}=.499 ; \mathrm{p}=.007$. However when grouping only IFR results, this effect was significantly attenuated. We suggest the need for future studies that clarify if participants employed a non spatial mnemonic strategy that obfuscated the influence of SC in IFR.

Overall these results have confirmed previous studies that found that the EM system associates each new experience within a spatiotemporal context and that this context becomes reactivated when the experience is recalled $[87,10,2]$. These results must be interpreted with caution as we are aware that a major topic of discussion for the validity of our results is the limited size of the sample.

Our AR framework proved to be adequate for analysis of the relation between space and memory because it helped evidence in a systematic, coherent and exhaustive way the structure and functioning of recall dynamics in both the guided and way-finding conditions. For the participants in the way-finding Condition using the AR system and the hand held device demonstrated to be an intuitive and straightforward task. Although our system accuracy needs further improvements, the overall dynamics and interactions with virtual contents are fluent and stable. Further improvements to the AR system and the handheld device could explore ways to device more precision in GPS readings and a greater refresh frequency for a more fluent interaction with the environment. Because of the use of current GPS technology this experiment can not take place indoors, which also could be a matter of research. Regarding the rendering qualities of the guided condition group we suggest further research must be done comparing the 


\section{References}

suitability of the realistic 3D virtual for our research needs, and compare to other rendering solutions such as to that of a viewpoint recording of the way-finders and its replaying over the guided condition.

We acknowledge the need to confirm these results by testing a greater participant population. An extension of this study may address the use of spatial mnemonic strategies, whether and how humans prefer spatial organizational strategies over temporal or categorical strategies. We also suggest further research on the role of encoding and retrieval modality [99] and the use of multi modal tasks. This field of research has interesting implications for the design of learning applications that could profit from spatial distribution of contents and exploratory learning behaviors. Our approach also presented potential benefits for memory studies and research on cerebral aging and neurodegenerative diseases. For instance, in Alzheimer's disease deficits in EM function are among the first cognitive symptoms observed and its study in ecologically valid tasks may be of profit. Furthermore, impaired EM function is also observed in a variety of other neuropsychiatric diseases including dissociative disorders, schizophrenia, and Parkinson's disease.

Taking a different approach, several recent fMRI studies compared recall of autobiographical memories with predicting possible personally relevant future events known as episodic future thinking [38], and found near complete overlap in the brain networks activated $[100,101]$. This scenario could provide ground for questioning the nature of SC in episodic future thinking. And also on the process of imagination as recent studies suggest that there are common processes in both EM and creative imagination, which can be characterized by the concept of scene construction [6]. If the Hippocampus plays a critical integrative role in a constructive process such as recollection it seems plausible that SC might also have a role in supporting the imagination, and in predicting the future [21].

1. D. Hume and P. H. Nidditch. David Hume: Enquiries Concerning Human Understanding and Concerning the Principles of Morals (Third Edition). OUP, Oxford UK, 1975.

2. P. Sederberg, M. Howard, and M. Kahana. A context-based theory of recency and contiguity in free recall. Psychological review, 115 4:893-912, 2008.

3. M. Howard and M. Kahana. A distributed representation of temporal context. Journal of Mathematical Psychology, 46:269-299, 2002.

4. L. Squire. "memory and the hippocampus: A synthesis from findings with rats, monkeys, and humans": Correction. Psychological Review, 99:582-582, 1992.

5. E. Maguire, N. Burgess, J. G. Donnett, R. Frackowiak, C. Frith, and J. O'Keefe. Knowing where and getting there: a human navigation network. Science, 280 5365:921-4, 1998.

6. D. Hassabis and E. Maguire. Deconstructing episodic memory with construction. Trends in Cognitive Sciences, 11:299-306, 2007.

7. D. Hassabis and E. Maguire. The construction system of the brain. Philosophical Transactions of the Royal Society B: Biological Sciences, 364:1263 - 1271, 2009. 
8. E. Maguire and S. Mullally. The hippocampus: A manifesto for change. Journal of Experimental Psychology. General, 142:1180 - 1189, 2013.

9. M. Bar (Editor). Predictions in the brain : using our past to generate a future. Oxford University Press, Oxford, UK, 2011.

10. M. Howard, M.S. Fotedar, A. V. Datey, and M. Hasselmo. The temporal context model in spatial navigation and relational learning: toward a common explanation of medial temporal lobe function across domains. Psychological review, 112 1:75$116,2005$.

11. M. Howard, M. Kahana, and A. Wingfield. Aging and contextual binding: Modeling recency and lag recency effects with the temporal context model. Psychonomic Bulletin \& Review, 13:439-445, 2006.

12. Sean M. Polyn, K. Norman, and M. Kahana. Task context and organization in free recall. Neuropsychologia, 47:2158-2163, 2009.

13. A. Pastor. Assessing the influence of spatial clustering in free recall. (Unpublished Master Thesis) Universitat Pompeu Fabra, 2015.

14. E. Tulving. Episodic and semantic memory. In E. Tulving and W. Donaldson, editors, Organization of memory. Academic Press, 1972.

15. T. Suddendorf and M. Corballis. Mental time travel and the evolution of the human mind. Genetic, social, and general psychology monographs, 123 2:133-67, 1997.

16. H. Eichenbaum, T. Otto, and N. Cohen. Two functional components of the hippocampal memory system. Behavioral and Brain Sciences, 17:449-472, 1994.

17. E. Tulving. Episodic memory: from mind to brain. Annual review of psychology, $53: 1-25,2002$.

18. D. Schacter, D. Addis, D. Hassabis, V. C. Martín, R. N. Spreng, and K. Szpunar. The future of memory: Remembering, imagining, and the brain. Neuron, 76:677694, 2012.

19. M. Howard and H. Eichenbaum. Time and space in the hippocampus. Brain Research, 1621:345-354, 2015.

20. D. Schiller, H. Eichenbaum, E. Buffalo, L. Davachi, D. Foster, S. Leutgeb, and C. Ranganath. Memory and space: Towards an understanding of the cognitive map. The Journal of Neuroscience, 35:13904 - 13911, 2015.

21. D. Hassabis, D. Kumaran, S. Vann, and E. Maguire. Patients with hippocampal amnesia cannot imagine new experiences. Proceedings of the National Academy of Sciences, 104:1726 - 1731, 2007.

22. E. Maguire and C. Frith. Aging affects the engagement of the hippocampus during autobiographical memory retrieval. Brain : a journal of neurology, $126 \mathrm{Pt}$ 7:1511-23, 2003.

23. T. Hafting, M. Fyhn, S. Molden, M. Moser, and E. Moser. Microstructure of a spatial map in the entorhinal cortex. Nature, 436:801-806, 2005.

24. F. Savelli, D. Yoganarasimha, and J. Knierim. Influence of boundary removal on the spatial representations of the medial entorhinal cortex. Hippocampus, 18 12:1270-82, 2008.

25. M. Hasselmo. A model of episodic memory: Mental time travel along encoded trajectories using grid cells. Neurobiology of Learning and Memory, 92:559-573, 2009.

26. R. Hayman and K. Jeffery. How heterogeneous place cell responding arises from homogeneous grids-a contextual gating hypothesis. Hippocampus, 18 12:1301-13, 2008. 
27. T. Shallice, P. Fletcher, C. Frith, P. Grasby, R. Frackowiak, and R. Dolan. Brain regions associated with acquisition and retrieval of verbal episodic memory. $\mathrm{Na}$ ture, 368:633-635, 1994.

28. C. Frith and R. Dolan. Brain mechanisms associated with top-down processes in perception. Philosophical transactions of the Royal Society of London. Series B, Biological sciences, 352 1358:1221-30, 1997.

29. C. Kirwan, J. Wixted, and L. Squire. Activity in the medial temporal lobe predicts memory strength, whereas activity in the prefrontal cortex predicts recollection. The Journal of Neuroscience, 28:10541 - 10548, 2008.

30. A. Lundervold, D. Wollschläger, and E. Wehling. Age and sex related changes in episodic memory function in middle aged and older adults. Scandinavian Journal of Psychology, 55:225 - 232, 2014.

31. F. Pauls, F. Petermann, and A. C. Lepach. Gender differences in episodic memory and visual working memory including the effects of age. Memory, 21:857 - 874, 2013.

32. A. Herlitz, E. Airaksinen, and E. Nordström. Sex differences in episodic memory: the impact of verbal and visuospatial ability. Neuropsychology, 13 4:590-7, 1999.

33. N. Persson, J. Wu, Q. Zhang, T. Liu, J. Shen, R. Bao, M. Ni, Y. Wang, and P. Spincemaille. Age and sex related differences in subcortical brain iron concentrations among healthy adults. NeuroImage, 122:385-398, 2015.

34. E. Tulving. Elements of episodic memory. Oxford University Press, 1983.

35. D. Schacter, D. Addis, and R. Buckner. Remembering the past to imagine the future: the prospective brain. Nature Reviews Neuroscience, 8:657-661, 2007.

36. E. Maguire and D. Hassabis. Role of the hippocampus in imagination and future thinking. Proceedings of the National Academy of Sciences, 108:E39 - E39, 2011.

37. R. Buckner. Prospection and the brain. Behavioral and Brain Sciences, 30:318319, 2007.

38. C. Atance and D. K. O'Neill. Episodic future thinking. Trends in Cognitive Sciences, 5:533-539, 2001.

39. C. Atance. Future thinking in young children. Current Directions in Psychological Science, 17:295 - 298, 2008.

40. C. Atance, S. Nur Celebi, S. Mitchinson, and C. E. V. Mahy. Thinking about the future: Comparing children's forced-choice versus "generative" responses in the "spoon test". Journal of experimental child psychology, 181:1-16, 2019.

41. T. Tsukiura, T. Fujii, T. Takahashi, R. Xiao, M. Sugiura, J. Okuda, T. Iijima, and A. Yamadori. Medial temporal lobe activation during context-dependent relational processes in episodic retrieval: An fmri study. Human Brain Mapping, 17:203-213, 2002.

42. K. Szpunar, J. Watson, and K. McDermott. Neural substrates of envisioning the future. Proceedings of the National Academy of Sciences, 104:642 - 647, 2007.

43. W. Scoville and B. Milner. Loss of recent memory after bilateral hippocampal lesions. Journal of Neurology, Neurosurgery \& Psychiatry, 20:11 - 21, 1957.

44. M. Mishkin. A memory system in the monkey. Philosophical transactions of the Royal Society of London. Series B, Biological sciences, 298 1089:83-95, 1982.

45. M. Mishkin, B. Malamut, and J. Bachevalier. Memories and habits: Two neural systems. In \& N. M. Weinberger G. Lynch, J. L. McGaugh, editor, Neurobiology of learning and memory. Guilford Press, New York, New York, USA, 1984.

46. M. Mishkin and H. L. Petri. Memories and habits: Some implications for the analysis of learning and retention. In L. R. Squire and N. Butters, editors, Neuropsychology of memory. Guilford Press, New York, New York, USA, 1984. 
47. L. Squire. Mechanisms of memory. Lancet, 1 7586:140-2, 1969.

48. L. Squire and S. Zola-Morgan. The medial temporal lobe memory system. Science, 253:1380 - 1386, 1991.

49. L. Nadel. A brain structure: the hippocampus. Science, 235 4796:1682a, 1987.

50. J. Aggleton and M. Brown. Episodic memory, amnesia, and the hippocampalanterior thalamic axis. The Behavioral and brain sciences, 22 3:425-44; discussion 444-89, 1999 .

51. H. Eichenbaum, A. Yonelinas, and C. Ranganath. The medial temporal lobe and recognition memory. Annual review of neuroscience, 30:123-52, 2007.

52. J. O'keefe and L. Nadel. The Hippocampus as a Cognitive Map. Oxford University Press, 1978.

53. N. Cohen and H. Eichenbaum. Memory, amnesia, and the hippocampal system. The MIT Press, 1993.

54. H. Eichenbaum and N. Cohen. From conditioning to conscious recollection: Memory systems of the brain. In Oxford psychology series; no. 35. Oxford University Press, 2001.

55. H. Intraub and M. Richardson. Wide-angle memories of close-up scenes. Journal of experimental psychology. Learning, memory, and cognition, 15 2:179-87, 1989.

56. S. Mullally, H. Intraub, and E. Maguire. Attenuated boundary extension produces a paradoxical memory advantage in amnesic patients. Current Biology, 22:261 $268,2012$.

57. M. J. Chadwick, S. Mullally, and E. Maguire. The hippocampus extrapolates beyond the view in scenes: An fmri study of boundary extension. Cortex; a Journal Devoted to the Study of the Nervous System and Behavior, 49:2067 2079, 2013.

58. K. S. Graham, M. Barense, and A. C. Lee. Going beyond ltm in the mtl: A synthesis of neuropsychological and neuroimaging findings on the role of the medial temporal lobe in memory and perception. Neuropsychologia, 48:831-853, 2010.

59. E. C. Tolman. Cognitive maps in rats and men. Psychological review, 55 4:189208,1948

60. P. W. Thorndyke. Distance estimation from cognitive maps. Cognitive Psychology, 13:526-550, 1981.

61. R. A. Epstein and N. Kanwisher. A cortical representation of the local visual environment. Nature, 392:598-601, 1998.

62. R. U. Muller, E. Bostock, J. Taube, and J. Kubie. On the directional firing properties of hippocampal place cells. In The Journal of neuroscience : the official journal of the Society for Neuroscience, 1994.

63. J. O'keefe and A. Speakman. Single unit activity in the rat hippocampus during a spatial memory task. Experimental Brain Research, 68:1-27, 2004.

64. A. Siegel and S. White. The development of spatial representations of large-scale environments. Advances in child development and behavior, 10:9-55, 1975.

65. A. Ekstrom and E. Isham. Human spatial navigation: representations across dimensions and scales. Current Opinion in Behavioral Sciences, 17:84-89, 2017.

66. A. P. Boone, X. Gong, and M. Hegarty. Sex differences in navigation strategy and efficiency. Memory \& Cognition, 46:909-922, 2018.

67. M. Fricke and O. Bock. Egocentric navigation is age-resistant: First direct behavioral evidence. Curr Neurobiol, 9(2):69-75, 2018.

68. I. Cherney, C. M. Brabec, and D. V. Runco. Mapping out spatial ability: Sex differences in way-finding navigation. Perceptual and Motor Skills, 107:747 - 760, 2008. 
69. B. Kuipers, N. Burgess, R. Epstein, V. Bingman, and F. Pazzaglia. Finding your way: The cognitive science of navigation. Cognitive Science, 33, 2011.

70. G. Groen, Arthur P. Wunderlich, M. Spitzer, R. Tomczak, and M. Riepe. Brain activation during human navigation: gender-different neural networks as substrate of performance. Nature Neuroscience, 3:404-408, 2000.

71. N. Burgess, E. Maguire, and J. O'Keefe. The human hippocampus and spatial and episodic memory. Neuron, 35:625-641, 2002.

72. J. O'keefe and J. Dostrovsky. The hippocampus as a spatial map. preliminary evidence from unit activity in the freely-moving rat. Brain research, 34 1:171-5, 1971.

73. G. Quirk, R. U. Muller, and J. Kubie. The firing of hippocampal place cells in the dark depends on the rat's recent experience. In The Journal of neuroscience : the official journal of the Society for Neuroscience, 1990.

74. L. T. Thompson and P. Best. Long-term stability of the place-field activity of single units recorded from the dorsal hippocampus of freely behaving rats. Brain Research, 509:299-308, 1990.

75. R. U. Muller, J. Kubie, and J. Ranck. Spatial firing patterns of hippocampal complex-spike cells in a fixed environment. In The Journal of neuroscience : the official journal of the Society for Neuroscience, 1987.

76. G. Quirk, R. U. Muller, J. Kubie, and J. Ranck. The positional firing properties of medial entorhinal neurons: description and comparison with hippocampal place cells. In The Journal of neuroscience : the official journal of the Society for Neuroscience, 1992.

77. L. Frank, E. Brown, and M. Wilson. Trajectory encoding in the hippocampus and entorhinal cortex. Neuron, 27:169-178, 2000.

78. D. C. Rowland, Y. Roudi, M. Moser, and E. Moser. Ten years of grid cells. Annual review of neuroscience, 39:19-40, 2016.

79. E. Moser, E. Kropff, and M. Moser. Place cells, grid cells, and the brain's spatial representation system. Annual review of neuroscience, 31:69-89, 2008.

80. R. Gardner, L. Lu, T. Wernle, M. Moser, and E. Moser. Correlation structure of grid cells is preserved during sleep. Nature Neuroscience, 22:598-608, 2019.

81. J. Taube, R. U. Muller, and J. Ranck. Head-direction cells recorded from the postsubiculum in freely moving rats. i. description and quantitative analysis. In The Journal of neuroscience : the official journal of the Society for Neuroscience, 1990.

82. J. Taube. Head direction cells. Scholarpedia, 4:1787, 2009.

83. F. Sargolini, M. Fyhn, T. Hafting, B. McNaughton, M. Witter, M. Moser, and E. Moser. Conjunctive representation of position, direction, and velocity in entorhinal cortex. Science, 312:758 - 762, 2006.

84. J. O'Keefe and N. Burgess. Geometric determinants of the place fields of hippocampal neurons. Nature, 381:425-428, 1996.

85. C. Lever, S. Burton, A. Jeewajee, J. O'keefe, and N. Burgess. Boundary vector cells in the subiculum of the hippocampal formation. The Journal of Neuroscience, 29:9771 - 9777, 2009.

86. C. MacDonald, K. Q. Lepage, U. Eden, and H. Eichenbaum. Hippocampal "time cells" bridge the gap in memory for discontiguous events. Neuron, 71:737-749, 2011.

87. J. Miller, E. Lazarus, S.M. Polyn, and M. Kahana. Spatial clustering during memory search. Journal of experimental psychology. Learning, memory, and cognition, 39 3:773-81, 2013. 
88. M. Kahana. Associative retrieval processes in free recall. Memory \& Cognition, 24:103-109, 1996.

89. J. R. Manning, S.M. Polyn, G. Baltuch, B. Litt, and M. Kahana. Oscillatory patterns in temporal lobe reveal context reinstatement during memory search. Proceedings of the National Academy of Sciences, 108:12893 - 12897, 2011.

90. L. Nyberg, R. Habib, A. McIntosh, and E. Tulving. Reactivation of encodingrelated brain activity during memory retrieval. Proceedings of the National Academy of Sciences of the United States of America, 97 20:11120-4, 2000.

91. M. Howard, I. Viskontas, K. H. Shankar, and I. Fried. Ensembles of human mtl neurons "jump back in time" in response to a repeated stimulus. Hippocampus, 22, 2012.

92. M. Kahana. Foundations of Human Memory. Oxford University Press, Oxford, UK, 2012.

93. J. M. Curiel and G. Radvansky. Mental organization of maps. Journal of Experimental Psychology: Learning, Memory and Cognition, 24:202-214, 1998.

94. Sean M. Polyn and M. Kahana. Memory search and the neural representation of context. Trends in Cognitive Sciences, 12:24-30, 2008.

95. F. J. Moreno-Martínez and P. R. Montoro. An ecological alternative to snodgrass \& vanderwart: 360 high quality colour images with norms for seven psycholinguistic variables. PLoS ONE, 7, 2012.

96. J. G. Snodgrass and M. Vanderwart. A standardized set of 260 pictures: norms for name agreement, image agreement, familiarity, and visual complexity. Journal of experimental psychology. Human learning and memory, 6 2:174-215, 1980.

97. E. Tulving. The effects of presentation and recall of material in free-recall learning. Journal of Verbal Learning and Verbal Behavior, 6:175-184, 1967.

98. Murdock and B. Bennet. The serial position effect of free recall. Journal of Experimental Psychology, 64:482-488, 1962.

99. B. Murdock and K. Walker. Modality effects in free recall. Journal of Verbal Learning and Verbal Behavior, 8:665-676, 1969.

100. T. Suddendorf and M. Corballis. New evidence for animal foresight? Animal Behaviour, 75:e1-e3, 2008.

101. K. Szpunar, D. Addis, Victoria C. McLelland, and D. Schacter. Memories of the future: New insights into the adaptive value of episodic memory. Frontiers in Behavioral Neuroscience, 7, 2013. 